\title{
The influence of climatic oscillations during the Quaternary Era on the genetic structure of Asian black bears in Japan
}

\author{
N Ohnishi ${ }^{1}$, R Uno ${ }^{2}$, Y Ishibashi ${ }^{3}$, HB Tamate ${ }^{4}$ and $\mathrm{T} \mathrm{Oi}^{1}$ \\ ${ }^{1}$ Kansai Research Center, Forestry and Forest Products Research Institute, Nagaikyutaro, Kyoto, Japan; ${ }^{2}$ Institute for Advanced \\ Biosciences, Keio University, Tsuruoka, Japan; ${ }^{3}$ Hokkaido Research Center, Forestry and Forest Products Research Institute, Sapporo, \\ Japan and ${ }^{4}$ Department of Biology, Faculty of Science, Yamagata University, Yamagata, Japan
}

\begin{abstract}
The Asian black bear (Ursus thibetanus) inhabits two of the main islands, Honshu and Shikoku, in Japan. To determine how climatic oscillations during the Quaternary Era affected the genetic structure of the black bear populations in Japan, we examined their phylogeographic relationships and compared their genetic structure. We analysed an approximately 700-bp sequence in the D-loop region of mitochondrial DNA collected from 589 bears in this study with 108 bears from a previous study. We observed a total of 57 haplotypes and categorized them into three clusters (Eastern, Western and Southern) based on the spatial distribution of the haplotypes. All but 2 of the 41 haplotypes in the Eastern cluster were distributed locally. Genetic diversity was generally low in northern Japan and high in central Japan.
\end{abstract}

Demographic tests rejected the expansion model in northern populations. Haplotypes of the Western and Southern clusters were unique to local populations. We conclude that the extant genetic structure of the Asian black bear populations arose as follows: first, populations became small and genetic drift decreased genetic diversity in the northern area during the last glacial period, whereas large continuous populations existed in the southern part of central Japan. These patterns were essentially maintained until the present time. In western and southern Japan, the effects of climatic oscillations were smaller, and thus, local structure was maintained.

Heredity (2009) 102, 579-589; doi:10.1038/hdy.2009.28; published online 25 March 2009

Keywords: female-biased philopatry; glacial periods; land bridge; phylogeography; vegetation shift

\section{Introduction}

Climatic oscillations during the Quaternary Era led to shifts in the distribution of animal and plant species. The extant species and their distributions are the result of adaptations to specific environments, migration or both factors (Hewitt, 2000). Phylogeographic studies based on molecular markers have revealed the relationships between intraspecific genetic variation and the geographic distributions of many plant and animal taxa, suggesting that glacial-interglacial oscillations have altered these distributions. During the last glacial period, broad-leaved trees were retained in refugia in the southern part of Honshu, the main island of Japan. In addition, the majority of the area that is currently broadleaved forest was then covered by coniferous forest in northern Honshu (Tsukada, 1982). It was only after the end of the last glacial period that the broad-leaved forest recovered and spread northward (Tsukada, 1982). The effects of this shift on the genetic structure of some broad-leaved tree populations have been revealed by

Correspondence. Current address: Dr N Ohnishi, Tohoku Research Center, Forestry and Forest Products Research Institute, Nabeyashiki, Morioka 020-0123, Japan.

E-mail: bigwest@affrc.go.jp

Received 17 July 2008; revised 9 February 2009; accepted 18

February 2009; published online 25 March 2009 phylogenetic studies. Specifically, the genetic diversity of species such as Japanese beech (Fagus crenata; Tomaru et al., 1998; Fujii et al., 2002), an endemic deciduous shrub (Stachyurus praecox; Ohi et al., 2003) and oak species (Quercus spp.; Kanno et al., 2004) is lower in the northern part of Honshu than in the southern part due to the founder effect that followed rapid postglacial expansion of these species.

It is possible that forest mammals shifted their distributions according to the changes in vegetation, particularly for vegetation that was crucial for their survival, and this would have affected their genetic structure similarly to what occurred in the abovementioned plant species. For example, Japanese macaques (Macaca fuscata) exhibit remarkably lower variability in mitochondrial DNA (mtDNA) in northern than in southern Honshu (Kawamoto et al., 2007). This suggests that the macaques rapidly expanded their distribution into the northern area during the recovery of the broad-leaved forests after the end of the last glacial period. Such a rapid expansion is also believed to have occurred in the Japanese giant flying squirrel (Petaurista leucogenys; Oshida et al., 2001).

The aim of this study was to determine the effect of climatic oscillations during the Quaternary Era on the genetic structure of Asian black bear (Ursus thibetanus) populations in Japan. This species is a medium-sized black bear that is distributed in southern and eastern 
Asia (Servheen, 1990). The black bear currently inhabits two of Japan's main islands, Honshu and Shikoku (Figure 1b), but it is thought to be extinct on Kyushu Island (Biodiversity Center of Japan, 2004). The present distribution of the black bear is generally continuous from northern to central Honshu, except in the northernmost peninsula, in contrast to the fragmented and small populations that inhabit western Japan.

The black bear is thought to have migrated from the Asian Continent to Japan during the glacial periods that occurred 0.5 and $0.3 \mathrm{My} \mathrm{BP}$ (Dobson and Kawamura, 1998). During these glacial periods, the Korean Peninsula was continuous with Kyushu Island; thus, black bears could cross the Korea-Kyushu land bridge. Following this immigration, interglacial and glacial periods occurred repeatedly; the last glacial period was between 80 and $12 \mathrm{ky}$ BP.

When considering the effect of climatic oscillations on the distribution of this species, we should focus on changes in the distribution of the broad-leaved forest, a

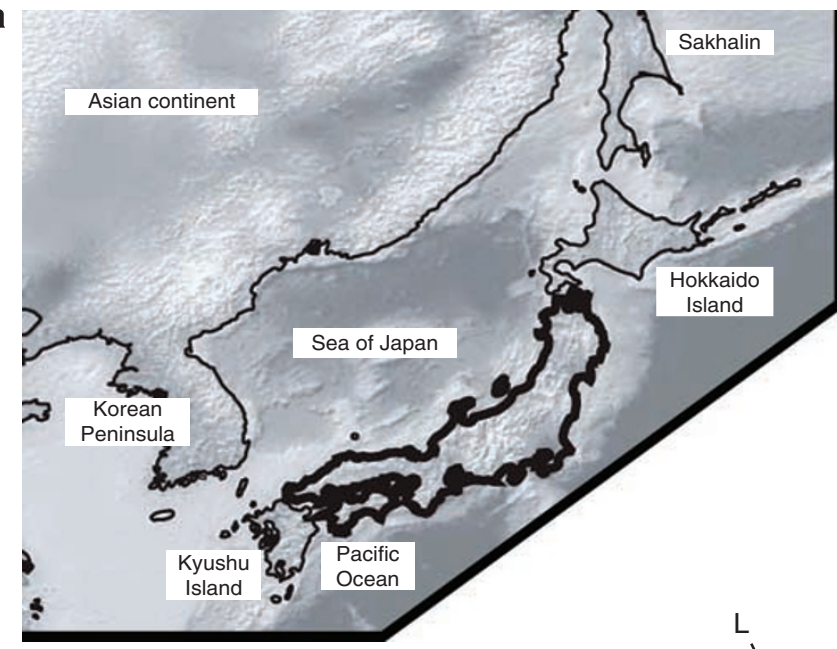

$\Lambda^{N}$ b

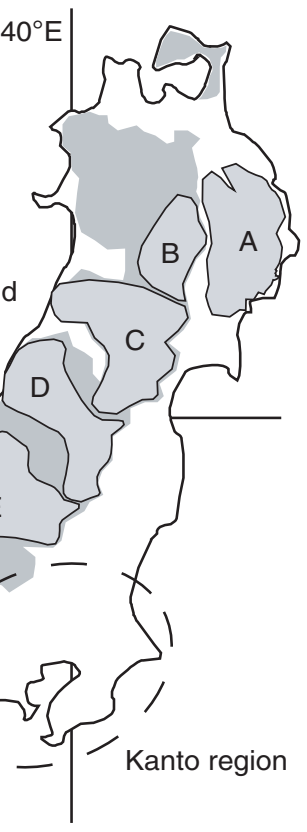

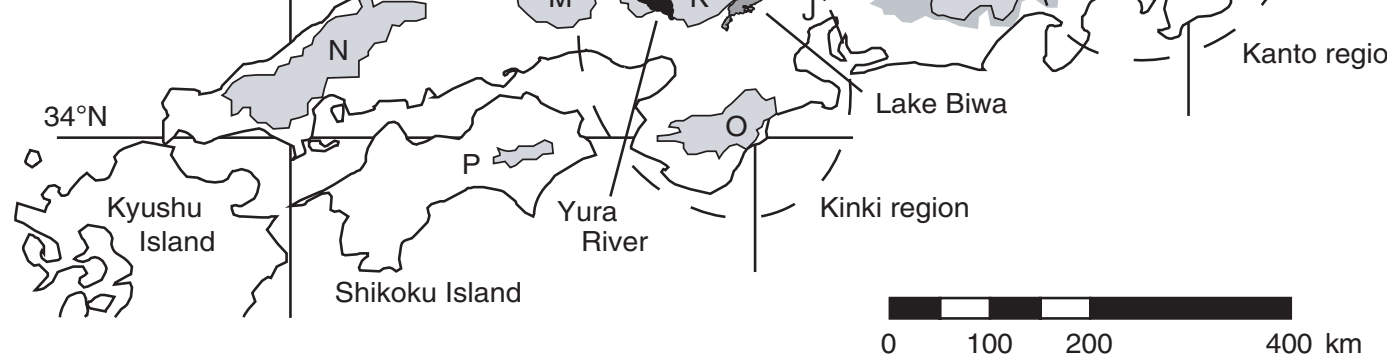

C
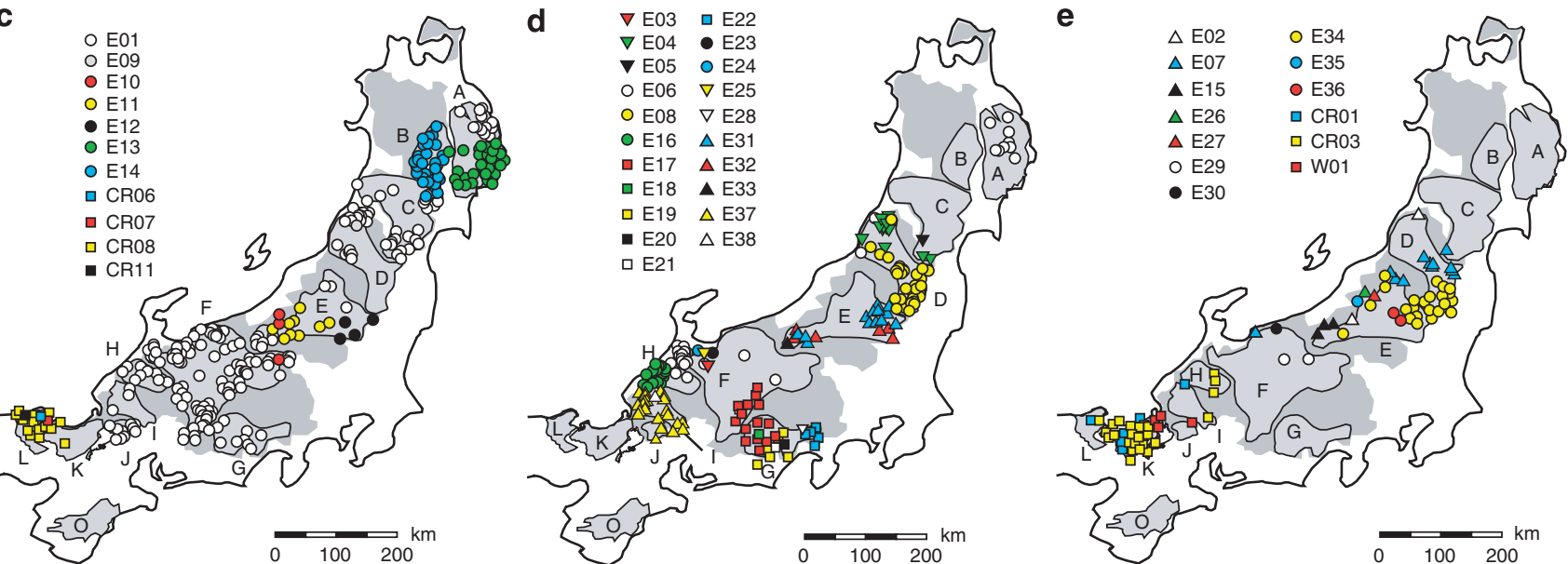

Figure 1 Maps of the study area and relevant adjacent regions: (a) altitudes and water depths of the Asian Continent near Japan, Japan and the areas between. The Korean Peninsula, the Sea of Japan, the Pacific Ocean and Sakhalin Island are indicated. (b) Distribution of the Asian black bear (Ursus thibetanus) in Japan (dark shading) and of the populations in this study (light shading). The Yura River, Lake Biwa, and the Kanto and Kinki regions are indicated: K, the eastern part of northern Kinki; L, the western part of northern Kinki; M, eastern Chugoku; N, the western Chugoku populations studied by Ishibashi and Saitoh (2004). (c-e) Points where black bears exhibiting the haplotypes in the A to L populations were captured: W01, E01 to E38 and CR01 to CR11 represent the UtCR-W01, UtCR-E and UtCR haplotypes, respectively. 
because the black bear prefers these habitats (Hashimoto et al., 2003). At present, except for the mountainous areas that are covered by coniferous forests that were planted for commercial use, the vegetation in western Japan is dominated by evergreen broad-leaved forest, which is thought to have replaced deciduous broad-leaved forests during glacial periods (Tsukada, 1982). Both forest types are suitable for the black bear. Shikoku and Kyushu were connected to Honshu, and the area that is currently underwater would also have been covered by deciduous broad-leaved forest during these glacial periods (Tsukada, 1982). Conversely, in eastern Japan, palynological studies of beech species (Minaki, 1996), on which the black bear depends strongly in its present distribution in northern Honshu (Oka et al., 2004), show that multiple refugia were sparsely located along the Pacific Ocean and the Sea of Japan south of approximately $38^{\circ} \mathrm{N}$ during the last glacial maximum (25-15 ky BP). At approximately $15 \mathrm{ky} \mathrm{BP}$, beech forests began to expand rapidly towards the north and the interior from these coastal refugia.

It can therefore be proposed that the black bear would have expanded northward with the recovery of the northern distribution of the beech forests. As a result, the genetic diversity of the black bear populations in the northern areas should be lower than those of the central areas because of founder effects, as was the case for the Japanese macaques and Japanese giant flying squirrels.

A previous phylogeographical study of black bears in western Honshu revealed that two distinct mtDNA lineages are separated by the Yura River in the Kinki region (Figure $1 \mathrm{~b}$ ) and that the western lineage contains various haplotypes (Ishibashi and Saitoh, 2004). The study suggested that three populations, L, M and $\mathrm{N}$ (WNK, EC and WC, respectively, in Ishibashi and Saitoh's study), became isolated from the K population (ENK in Ishibashi and Saitoh's study) on the eastern side of the river, resulting in two populations that retreated to different refugia during the last glacial period. A similar phylogeographic pattern has been observed in other large mammal species such as the Japanese macaque (Kawamoto et al., 2007), Japanese wild boar (Sus scrofa; Watanobe et al., 2003) and sika deer (Cerous nippon; Tamate et al., 1998; Nagata et al., 1999), all of which exhibit two mtDNA lineages, with the distributions of the haplotypes of each lineage separated in the Kinki region. Given these data, Ishibashi and Saitoh (2004) suggested that the black bear would also have two Japanese lineages, separated at the Yura River.

Taking the moderate shift of the broad-leaved forests into consideration, it is likely that the black bear was widely distributed throughout western Japan during the glacial periods. As a result, genetic diversity within western Japan has likely remained relatively high, which means that the $\mathrm{P}$ population should exhibit a genetic structure similar to that of the western Honshu populations.

\section{Materials and methods}

DNA samples

DNA samples were taken from a total of 589 bears: 560 bears from continuous populations east of Lake Biwa, 15 from the $\mathrm{K}$ population, 9 from the $\mathrm{O}$ population and 5 from the P population (Figure 1b). Tissue, blood and hair samples were collected from bears that were hunted or captured for pest control between 1991 and 2007. In the P population, hairs were collected using hair traps between 2003 and 2005. One sample of faeces was collected from the $\mathrm{O}$ population in 2006.

As the borders between populations are not known for the continuous populations east of Lake Biwa, we predefined 10 populations (A-J; Figure 1) using Geneland 2.0.12 (Guillot et al., 2005) with geographical information (that is, the locations at which individuals were sampled), as well as haplotype data that were obtained in this study following the approach of Coulon et al. (2006).

We obtained data for 108 additional bears from Ishibashi and Saitoh (2004): $n=28$ of 32 for the K population, $n=25$ of 32 for the $\mathrm{L}$ population and all data for the $\mathrm{M}(n=22)$ and $\mathrm{N}(n=33)$ populations. The data from that previous study for the $\mathrm{K}$ and $\mathrm{L}$ populations were selected based on the availability of location data for the capture points.

\section{DNA extraction and sequencing}

Genomic DNA was extracted from tissue and blood using the standard Proteinase $\mathrm{K} /$ phenol/chloroform method (Sambrook et al., 1989) or MagExtractor (Toyobo, Osaka, Japan), from hairs using the QIAmp DNA Mini Kit (QIAGEN K. K., Tokyo, Japan) or the ChargeSwitch Forensic DNA Purification Kit (Invitrogen) and from faeces using the QIAmp DNA Stool Mini Kit (QIAGEN K. K.).

A highly variable region of the mtDNA control region and its $5^{\prime}$-flanking region (approximately $700 \mathrm{bp}$, from 702 to $706 \mathrm{bp}$ ) were sequenced as described by Ishibashi and Saitoh (2004), with some modifications. Three primers (CB-Z, BED1 and D4) used by Ishibashi and Saitoh (2004) were replaced with UT-5 (5'-TACCTTGGTC TTGTAAGC-3'), UT-6 (5'-AAGACTCAAGGAAGAAGC AA-3') and UT-7 (5'-CATGTTTGCGCGTGCATTTAGTC TTG-3'), respectively, and the annealing temperature was $50{ }^{\circ} \mathrm{C}$ in the PCR amplification. The partial mtDNA sequence was determined using a DYEnamics ET Terminator Cycle Sequencing Kit (Amersham Pharmacia Biotech, Little Chalfont, Buckinghamshire, UK) using a 3100-Avant Genetic Analyzer (Applied Biosystems, Carlsbad, CA, USA). The previous study identified a T-repeat site in the mtDNA control region in which the number of Ts varied (Ishibashi and Saitoh, 2004). Given that the deduced mutation process for a repeat-number variation in short-tandem repeats differs from that for a base-incorporation error (Eisen, 1999), the T-repeat variation was not taken into account in this study.

Phylogenetic analyses and estimation of divergence time Genealogical relationships among the observed mtDNA haplotypes were inferred using four haplotypes of the black bear observed on the Asian continent and Taiwan: DQ402478, Sichuan Province, China (Hou et al., 2007); EF196661, Yunnan Province, China (Yu et al., 2007); EF667005, northern Korea (Hwang et al., 2008); EF076773, Taiwan (Tsai et al., 2009). A haplotype of the brown bear (Ursus arctos) on Hokkaido, the northernmost island of Japan (Matsuhashi et al., 1999, 2001), was used as the outgroup (EMBL/GenBank/DDBJ: AB013043). Bayesian phylogenetic and maximum-likelihood (ML) analyses 
were conducted using BEAST 1.4.7 (Drummond and Rambaut, 2007) and Treefinder (Jobb et al., 2004), respectively. The TIM $+\mathrm{I}+\mathrm{G}$ model was determined to be an appropriate model of nucleotide evolution for these analyses based on the value of Akaike's information criterion in MODELTEST 3.7 (Posada and Crandall, 1998). Posterior distributions of parameters were approximated using two independent Markov-chain Monte Carlo analyses of 30000000 steps each, following a discarded burn-in of 3000000 steps for the Bayesian phylogenetic analysis. Samples from the two chains that yielded similar results were combined. A maximum clade credibility tree was drawn using TreeAnnotator 1.4.7 in BEAST. The ML analysis with the TIM $+\mathrm{I}+\mathrm{G}$ model was performed using the likelihood-ratchet method (Nixon, 1999; Vos, 2003). The branch statistical support was obtained after 1000 bootstrap replicates. The statistical parsimony network was constructed using TCS $1.18 \mathrm{~m}$ software (Clement et al., 2000).

The time to the most recent common ancestor $\left(t_{\mathrm{MRCA}}\right)$ for each pair of lineages of the black bear was estimated using BEAST 1.4.7. We adopted the following assumed divergence times as calibration points: 2.0 and $3.5 \mathrm{My} \mathrm{BP}$ between the black bear of the Asian continent and the brown bear (Waits et al., 1999), and 0.5 My BP among all lineages of the black bear in Japan (Dobson and Kawamura, 1998). Other parameters were the same as for the phylogenetic analysis.

\section{Hierarchical analysis of molecular variance}

A hierarchical analysis of molecular variance (AMOVA; Excoffier et al., 1992) was performed to reveal the geographical structure of genetic variations using Arlequin 3.1 (Excoffier et al., 2005). In this procedure, standard variance components and $\Phi$-statistics were computed at each of three levels of population subdivision: among geographical areas, among populations within areas and within populations. The genetic distance between mtDNA haplotypes was estimated using the proportion-of-differences method without $\gamma$ correction. The significance of the variance components and of the $\Phi$-statistics was tested using 10000 random permutations (Excoffier et al., 1992). In this analysis, we defined three geographic areas based on the observed phylogeographic breaks: Southern, Western and Eastern cluster areas (see the Results section).

Genetic diversity and population demographic indices Several genetic diversity indices were estimated for each of the populations using Arlequin: gene diversity $(H)$, the mean number of pairwise nucleotide differences $(d)$ and nucleotide diversity between all pairs of sequences $(\pi)$. Relationships between geographical information (longitude and latitude) and the $H$ and $d$ values of each population east of Lake Biwa were analysed using a bubble chart. Tajima's D statistic (Tajima, 1989) and Fu's $F_{S}$ statistic $(\mathrm{Fu}, 1997)$ were calculated using Arlequin to test for population expansion. A mismatch distribution (Li, 1977; Slatkin and Hudson, 1991; Rogers and Harpending, 1992; Schneider and Excoffier, 1999) was also constructed using Arlequin to examine the recent demographic history of each population. The deviation from the sudden population model was tested using Harpending's raggedness index $(r)$.

\section{Results}

We observed 43 novel haplotypes in the 589 bear samples from continuous populations east of the Yura River, and two isolated populations ( $\mathrm{O}$ and $\mathrm{P})$, by sequencing the approximately 700-bp mtDNA control region. Therefore, when the 14 previously identified UtCR-haplotypes from 108 bears (Ishibashi and Saitoh, 2004) were included, a total of 57 haplotypes were observed in the 697 black bears. Base substitutions were observed at 42 variable positions in the 43 novel haplotypes, and at 48 variable positions in all 57 haplotypes. These haplotype sequences were deposited in the EMBL/GenBank/DDBJ Nucleotide Sequence Database (UtCR-E01 to UtCR-E38, AB441772 to AB441809; UtCR-S01 to UtCR-S04, AB441810 to AB441813; UtCR-W01, AB441814).

\section{Genealogical analyses}

The Bayesian phylogenetic analysis revealed three major clusters of black bears in Japan based on the relationships among the haplotypes (Figure 2a). UtCR-01 plus UtCR-03, and UtCR-06 to UtCR-20 were structured into two different clusters in the previous study (Ishibashi and Saitoh, 2004), but they were included in a single cluster in our analysis. In addition, we identified UtCRW01, which was included in a cluster with UtCR-01 and UtCR-03. The UtCR-01 to UtCR-20 and UtCR-W01 haplotypes were mainly observed west of Lake Biwa (Table 1; Figures 1c and e). The two haplotypes from the $\mathrm{P}$ population (UtCR-S01 and UtCR-S02) formed another cluster with the two haplotypes from the $\mathrm{O}$ population (UtCR-S03 and UtCR-S04). The haplotypes in the third cluster were observed east of Lake Biwa (Figures 1c-e). UtCR-E01 was identified in 197 of the 560 samples from east of Lake Biwa (Table 1) and was widely distributed from Lake Biwa to northeastern Honshu (Figure 1c). UtCR-E06 was also found throughout a large area (Figure 1d), whereas other haplotypes were generally observed primarily in local areas. From these results, we designated three clusters: the Western, Southern and Eastern clusters. In addition, we designated the two areas to the east and west of Lake Biwa as the Eastern cluster area and the Western cluster area, respectively. The $\mathrm{O}$ and $\mathrm{P}$ populations were collectively named the Southern cluster area. The four sequences obtained from the black bears on the Asian continent and Taiwan showed higher divergences than the three Japanese clusters.

The Southern cluster (UtCR-S01 to UtCR-S04) was also categorized as a single cluster by the ML analysis (Figure 2b). The Eastern haplotypes (UtCR-E01 to UtCR-E38) formed a single minor cluster, even though the Western and Eastern clusters were less distinct in this analysis than in the Bayesian analysis.

The haplotype network calculated by statistical parsimony also suggested the existence of three large clusters (Figure 3), as had been demonstrated by the Bayesian phylogenetic tree (Figure 2a). The three clusters were connected to each other by unsampled or extinct haplotypes; in particular, the Southern cluster was strongly separated from the Western cluster (by four missing haplotypes). The network structure in the Eastern cluster was much more complex than those in the other two clusters, and some subgroups of the 
a

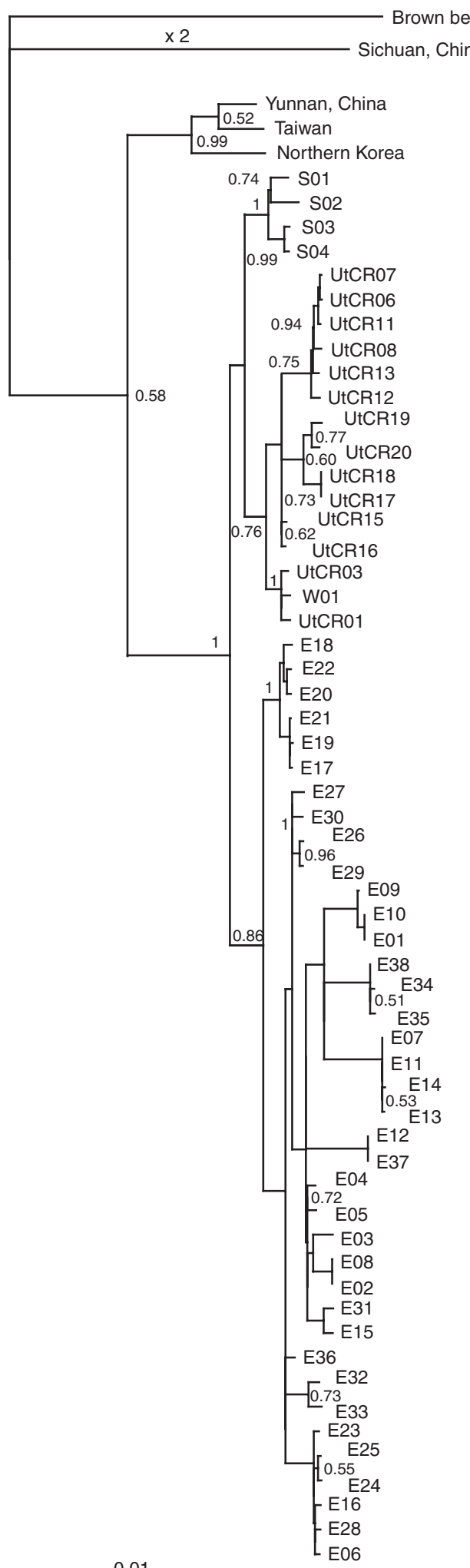

b

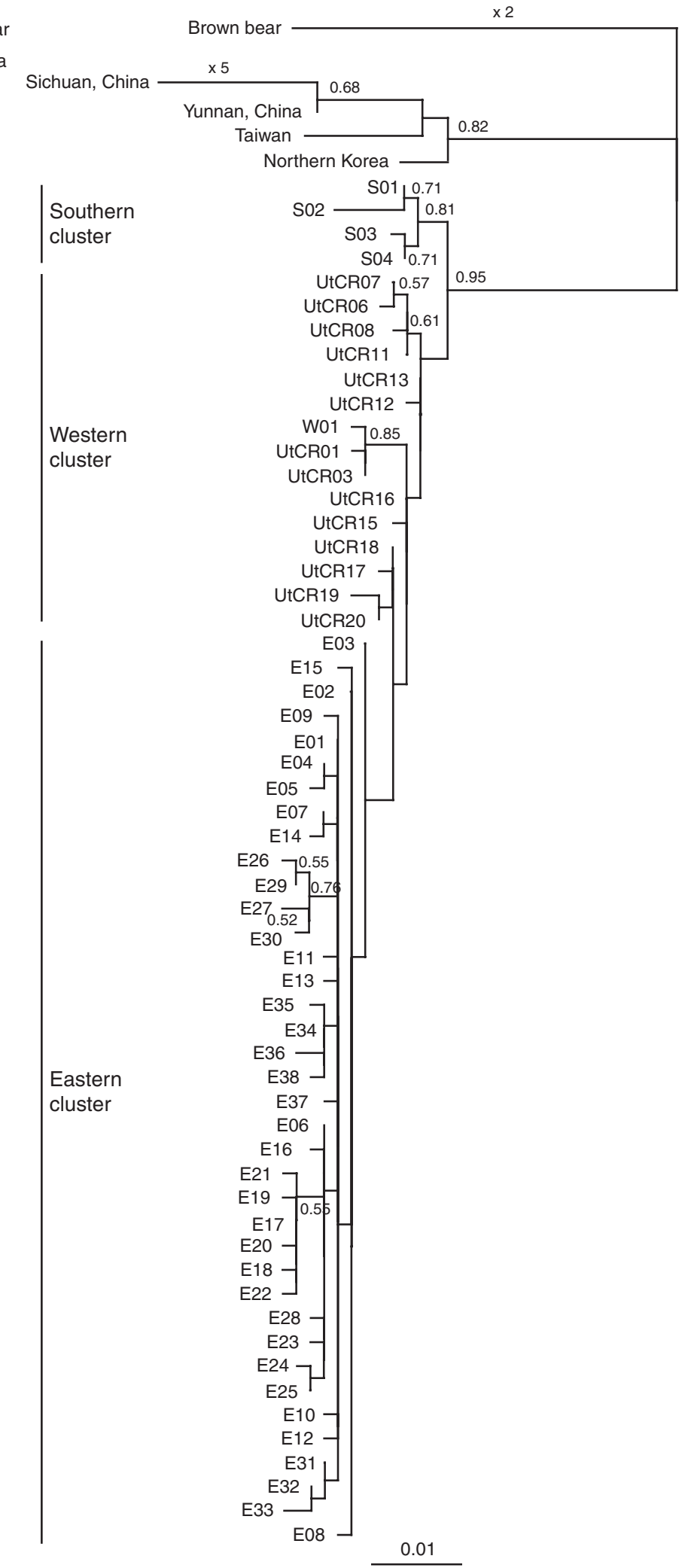

Figure 2 (a) Maximum clade credibility tree for the observed mitochondrial DNA (mtDNA) control region sequences created using Bayesian analysis. Only posterior probabilities greater than 50\% are shown. (b) Maximum-likelihood tree for the observed mtDNA control region sequences. Only bootstrap values greater than 50\% are shown. W01, S01 to S04 and E01 to E38 represent the UtCR-W01, UtCR-S01 to UtCRS04 and UtCR-E01 to UtCR-E38 haplotypes, respectively. Data for the UtCR series of haplotypes was obtained from Ishibashi and Saitoh (2004). Four mtDNA sequences of the Asian black bear were obtained from China's Sichuan Province (Hou et al., 2007) and Yunnan Province (Yu et al., 2007), from northern Korea (Hwang et al., 2008) and from Taiwan (Tsai et al., 2009). The mtDNA sequences of the brown bear (Ursus arctos) in Japan (Matsuhashi et al., 1999, 2001) were used as an outgroup. 
Table 1 Genetic diversity indices and the number of observed haplotypes in Asian black bear populations

\begin{tabular}{|c|c|c|c|c|c|c|c|c|c|c|c|c|c|c|c|c|c|c|c|c|c|c|c|c|c|c|c|c|c|c|}
\hline \multirow[t]{2}{*}{ Population } & \multirow[t]{2}{*}{$\mathrm{n}$} & \multirow[t]{2}{*}{$\mathrm{k}$} & \multirow[t]{2}{*}{$S$} & \multirow[t]{2}{*}{$\mathrm{H}$} & \multirow[t]{2}{*}{ d } & \multirow[t]{2}{*}{$\pi\left(\times 10^{3}\right)$} & \multicolumn{3}{|c|}{ Demographic expansion } & \multicolumn{21}{|c|}{ Haplotypes } \\
\hline & & & & & & & $F u^{\prime} s F_{S}$ & ajima's $D$ & $\mathrm{r}$ & E01 & E02 & E03 & E04 & E05 & E06 & E07 & E08 & E09 & E10 & E11 & E12 & E13 & E14 & E15 & E16 & E17 & E18 & E19 & E20 & E21 \\
\hline & 67 & 3 & 2 & 858 & 6730 & 953 & 24 & 1.01 & 0.09 & 12 & - & - & - & - & 9 & - & - & - & - & - & - & 46 & - & - & - & - & - & - & - & - \\
\hline & 31 & 3 & 2 & 2882 & 5118 & & 0.13 & 0.05 & $0.49^{*}$ & 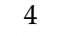 & - & - & - & - & - & - & - & - & - & - & - & 1 & 26 & - & - & - & - & - & - & - \\
\hline & 34 & 5 & 4 & 09 & 1456 & & 2.7 & -1.35 & 0.24 & 28 & - & - & 3 & 1 & - & 1 & - & - & - & - & - & - & 1 & - & - & - & - & - & - & - \\
\hline D & 113 & 10 & 8 & 0.7930 & 1.7291 & & -1.23 & 0.35 & $0.13^{*}$ & 21 & 1 & 12 & 10 & - & 2 & 9 & 42 & 1 & - & - & 1 & - & - & - & - & - & - & - & - & - \\
\hline E & 69 & 15 & 16 & 0.8875 & 1.9425 & 2 & $-6.04^{* *}$ & -1.10 & $0.10^{* *}$ & 10 & 1 & - & - & - & - & 3 & - & - & 2 & 9 & 3 & - & - & 3 & - & - & - & - & - & - \\
\hline & 127 & 11 & 12 & 4 & 3719 & & ** & $-1.56^{*}$ & 0.12 & 90 & - & 1 & - & - & 19 & 1 & - & - & 1 & - & - & - & - & - & - & 9 & - & - & - & - \\
\hline G & 42 & 8 & 9 & 0.7828 & 3386 & & -0.99 & -0.35 & 0.08 & 9 & - & - & - & - & - & - & - & - & - & - & - & - & - & - & - & 15 & 1 & 9 & 1 & 1 \\
\hline $\mathrm{H}$ & 31 & 4 & 7 & 0.6516 & 1.8452 & & 2.21 & & 0.20 & 9 & - & - & - & - & 3 & - & - & - & - & - & - & - & - & - & 16 & - & - & - & - & - \\
\hline & & 6 & 10 & 0 & 1 & & -0 . & -1.3 & & 3 & - & - & - & - & - & - & - & - & - & - & - & - & - & - & 2 & - & - & - & - & - \\
\hline & 14 & 3 & 8 & 0.3846 & 1.2640 & & 1.3 & $-1.88^{*}$ & 0.22 & 11 & - & - & - & - & - & - & - & - & - & - & - & - & - & - & - & - & - & - & - & - \\
\hline K & 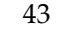 & 4 & 7 & 0.4009 & 1.1830 & & 0. & -1.46 & 0.18 & - & - & - & - & - & - & - & - & - & - & - & - & - & - & - & - & - & - & - & - & - \\
\hline & & 6 & 8 & 0.3667 & 1.2800 & & -1. & -1.2 & & - & - & - & - & - & - & - & - & - & - & - & - & - & - & - & - & - & - & - & - & - \\
\hline $\mathrm{N} \mathbf{1}$ & 22 & 3 & 3 & 0.6104 & 0.9481 & & 1.19 & 0.39 & 0.0 & - & - & - & - & - & - & - & - & - & - & - & - & - & - & - & - & - & - & - & - & - \\
\hline $\mathrm{N}$ & 33 & 6 & 7 & 0.5038 & 1.3106 & 1.856 & -0.72 & -0.69 & 0.13 & - & - & - & - & - & - & - & - & - & - & - & - & - & - & - & - & - & - & - & - & - \\
\hline 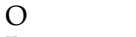 & 9 & 2 & 1 & 0.5000 & 000 & & & & & - & - & - & - & - & - & - & - & - & - & - & - & - & - & - & - & - & - & - & - & - \\
\hline 1 & 5 & 3 & 11 & 0.3667 & 5.4000 & 7.64 & 2.46 & 0.16 & 0.36 & - & - & - & - & - & - & - & - & - & - & - & - & - & - & - & - & - & - & - & - & - \\
\hline Total & 697 & 57 & 48 & 0.8967 & 3.3850 & 4.795 & & & & 197 & 2 & 13 & 13 & 1 & 33 & 14 & 42 & 1 & 3 & 9 & 4 & 47 & 27 & 3 & 18 & 24 & 1 & 9 & 1 & 1 \\
\hline
\end{tabular}

Haplotypes

E22 E23 E24 E25 E26 E27 E28 E29 E30 E31 E32 E33 E34 E35 E36 E37 E38 W01 CR01 CR03 CR06 CR07 CR08 CR11 CR12 CR13 CR15 CR16 CR17 CR18 CR19 CR20 S01 S02 S03 S04

\begin{tabular}{|c|c|c|c|c|c|c|c|c|c|c|c|c|c|c|c|c|c|c|c|c|c|c|c|c|c|c|c|c|c|c|c|c|c|c|c|c|}
\hline & - & - & - & - & - & - & - & - & - & - & - & - & - & - & - & - & - & - & - & - & - & - & - & - & - & - & - & - & - & - & - & & & - & - & -- \\
\hline & - & - & - & - & - & - & - & - & - & - & - & - & - & - & - & - & - & - & - & - & - & - & - & - & - & - & - & - & - & - & - & & & - & - & -- \\
\hline & - & - & - & - & - & - & - & - & - & - & - & - & - & - & - & - & - & - & - & - & - & - & - & - & - & - & - & - & - & - & - & - & & - & - & -- \\
\hline & - & - & - & - & - & - & - & - & - & - & - & - & 14 & - & - & - & - & - & - & - & - & - & - & - & - & - & - & - & - & - & - & & & - & - & - \\
\hline & - & - & - & - & 1 & 1 & - & - & - & 14 & 6 & 1 & 12 & 1 & 2 & - & - & - & - & - & - & - & - & - & - & - & - & - & - & - & - & - & & - & - & -- \\
\hline & - & 1 & 1 & 1 & - & - & - & 2 & 1 & - & - & - & - & - & - & - & - & - & - & - & - & - & - & - & - & - & - & - & - & - & - & - & & - & - & - \\
\hline & 5 & - & - & - & - & - & 1 & - & - & - & - & - & - & - & - & - & - & - & - & - & - & - & - & - & - & - & - & - & - & - & - & - & & - & - & - \\
\hline & - & - & - & - & - & - & - & - & - & - & - & - & - & - & - & - & - & - & - & 3 & - & - & - & - & - & - & - & - & - & - & - & - & & - & - & - \\
\hline & - & - & - & - & - & - & - & - & - & - & - & - & - & - & - & 24 & 1 & - & 1 & 1 & - & - & - & - & - & - & - & - & - & - & - & - & & - & - & - \\
\hline & - & - & - & - & - & - & - & - & - & - & - & - & - & - & - & 2 & - & 1 & - & - & - & - & - & - & - & - & - & - & - & - & - & _ & & - & - & -- \\
\hline & - & - & - & - & - & - & - & - & - & - & - & - & - & - & - & - & - & 4 & 4 & 33 & - & - & 2 & - & - & - & - & - & - & - & - & & & - & - & -- \\
\hline & - & - & - & - & - & - & - & - & - & - & - & - & - & - & - & - & - & - & 1 & 1 & 1 & 1 & 20 & 1 & - & - & - & - & - & - & - & - & & - & - & -- \\
\hline & - & - & - & - & - & - & - & - & - & - & - & - & - & - & - & - & - & - & - & - & - & - & 3 & - & - & - & - & - & 7 & 12 & - & _ & & - & - & - \\
\hline 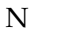 & - & - & - & - & - & - & - & - & - & - & - & - & - & - & - & - & - & - & - & - & - & - & - & - & 1 & 23 & 1 & 4 & - & - & 3 & & & - & - & - \\
\hline & - & - & - & - & - & - & - & - & - & - & - & - & - & - & - & - & - & - & - & - & - & - & - & - & - & - & - & - & - & - & - & - & & - & - & 63 \\
\hline & - & - & - & - & - & - & - & - & - & - & - & - & - & - & - & - & - & - & - & - & - & - & - & - & - & - & - & 1 & - & - & - & & & & & - \\
\hline otal & 5 & 1 & 1 & 1 & 1 & 1 & 1 & 2 & 1 & 14 & 6 & 1 & 26 & 1 & 2 & 26 & 1 & 5 & 6 & 38 & 1 & 1 & 25 & 1 & 1 & 23 & 1 & 5 & 7 & 12 & 3 & 1 & & 2 & 2 & 63 \\
\hline
\end{tabular}

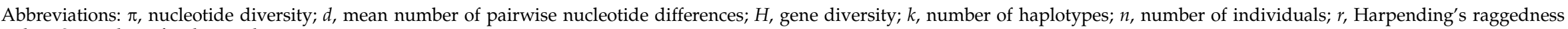
index; $S$, number of polymorphic sites.

W01, S01 to S04 and E01 to E38 represent UtCR-W01, UtCR-S01 to UtCR-S04 and UtCR-E01 to UtCR-E38, respectively. CR represent the UtCR values from Ishibashi and Saitoh (2004).

${ }^{*}$ and ${ }^{* *}$ in the demographic expansion estimators indicate significant values $P<0.05$ and $P<0.02$, respectively. 


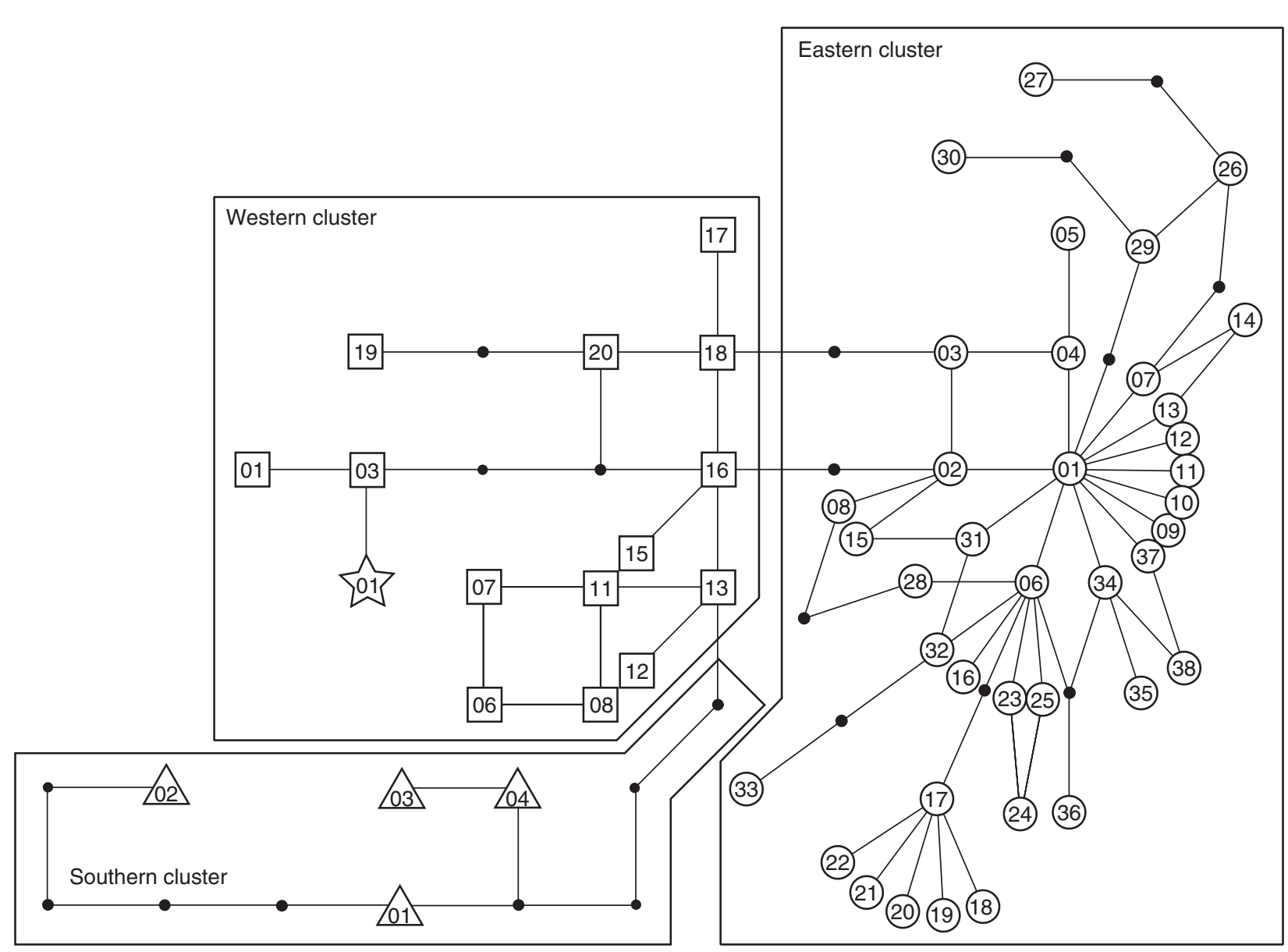

Figure 3 Statistical parsimony network for the mitochondrial DNA (mtDNA) control region sequences. Each line in the network represents a single mutational change. Triangles, large circles and squares represent the UtCR-S, UtCR-E and UtCR haplotypes, respectively. Data for the UtCR haplotypes were taken from Ishibashi and Saitoh (2004). The star represents the UtCR-W01 haplotype. Small black circles indicate interior nodes that were absent from the sample or that are extinct haplotypes. The thick lines group the haplotypes into three regional clusters.

Table 2 The time to the most recent common ancestor $\left(t_{\mathrm{MRCA}} \pm\right.$ s.d.) estimated from the coalescence tree

\begin{tabular}{|c|c|c|c|c|}
\hline \multirow[t]{2}{*}{ The split } & \multicolumn{2}{|c|}{$\mathrm{t}_{B / B}=2.0 \mathrm{MyBP}$} & \multicolumn{2}{|c|}{$\mathrm{t}_{B / B}=3.5 \mathrm{My} \mathrm{BP}$} \\
\hline & $\mathrm{t}_{J}=0.5 \mathrm{MyBP}$ & $\mathrm{t}_{J}=$ unknown & $\mathrm{t}_{J}=0.5 \mathrm{My} \mathrm{BP}$ & $\mathrm{t}_{J}=$ unknown \\
\hline Asia/Japan ${ }^{\mathrm{a}}$ & $1.833( \pm 0.0016)$ & $1.417( \pm 0.0284)$ & $2.405( \pm 0.0221)$ & $2.565( \pm 0.0603)$ \\
\hline Western/Southern ${ }^{\mathrm{b}}$ & $0.447( \pm 0.0011)$ & $0.297( \pm 0.0066)$ & $0.448( \pm 0.0009)$ & $0.540( \pm 0.0143)$ \\
\hline Western/Eastern ${ }^{\mathrm{b}}$ & $0.431( \pm 0.0019)$ & $0.295( \pm 0.0066)$ & $0.458( \pm 0.0011)$ & $0.537( \pm 0.013$ \\
\hline Southern/Eastern ${ }^{\mathrm{b}}$ & $0.480( \pm 0.0005)$ & $0.320( \pm 0.0073)$ & $0.483( \pm 0.0004)$ & $0.583( \pm 0.0151)$ \\
\hline
\end{tabular}

$t_{\mathrm{B} / \mathrm{B}}$ is assumed to represent the divergence time of the Asian black bear (Ursus thibetanus) on the Asian continent and the brown bear (U. arctos), and $t_{\mathrm{J}}$ is assumed to be the divergence time of the black bear in Japan.

${ }^{\mathrm{a}} t_{\text {MRCA }}$ for the black bear lineages between the Asian continent and Japan.

$\mathrm{b}_{t_{\mathrm{MRCA}}}$ for the black bear lineages among the Southern, Western and Eastern clusters.

haplotypes have diverged radially from UtCR-E01, UtCR-E06 and UtCR-E17.

The times to the most recent common ancestor $\left(t_{\mathrm{MRCA}}\right)$ for the lineages of black bears from the Asian continent and Japan, and among the three clusters in Japan, were estimated using assumed values of 2.0 and $3.5 \mathrm{My}$ BP for the brown and black bears, respectively, and $0.5 \mathrm{My} \mathrm{BP}$ for the three Japanese clusters (Table 2). When calculated with an unknown $t_{\mathrm{MRCA}}$ for Japan, the $t_{\mathrm{MRCA}}$ values for all three clusters differed greatly between $t_{\mathrm{MRCA}}$ of 2.0 and 3.5 My BP for the brown and black bears. However, when a $t_{\text {MRCA }}$ value for Japan of $0.5 \mathrm{My} \mathrm{BP}$ was assumed, the $t_{\mathrm{MRCA}}$ values for all three Japanese clusters were between 0.431 and $0.483 \mathrm{My} \mathrm{BP}$, and the $t_{\mathrm{MRCA}}$ values for the Asian and Japanese lineages were 1.833 and $2.405 \mathrm{My}$ $\mathrm{BP}$; the divergence times of the brown and black bears were estimated to be 2.0 and $3.5 \mathrm{My} \mathrm{BP}$, respectively.

\section{Hierarchical analysis of molecular variance}

The hierarchical AMOVA showed that the largest fraction of molecular genetic variance $(57.0 \%)$ was 
Table 3 Hierarchical analysis of molecular variance (AMOVA) for the mtDNA sequences of the samples collected from 697 black bears

\begin{tabular}{lccccc}
\hline Variance component & Variance & Total \% & $\Phi$ & $P$ \\
\hline Among areas & 1.62 & 57.00 & 0.57 & $<0.0001$ \\
Among populations within areas & 0.60 & 20.92 & 0.49 & $<0.0001$ \\
Within populations & 0.63 & 22.08 & 0.78 & $<0.0001$ \\
\hline
\end{tabular}

found among areas (Table 3). The remainder of the molecular variance was divided roughly equally between the other two hierarchical levels, at 20.9\% (among populations within an area) and 22.1\% (within populations). The $\Phi$-statistics for all three hierarchical levels were significantly greater than zero $(P<0.0001)$, but the value was highest within populations $\left(\Phi_{\mathrm{ST}}=0.78\right)$. These results indicate that all three hierarchical levels contributed significantly to the overall genetic variation.

\section{Genetic diversity and demographic expansion}

The parameters representing the genetic diversities of the populations are listed in Table 1. Figure 4 shows a bubble chart of the relationships between geographical information (longitude and latitude) and the gene $(H)$ and nucleotide $(\pi)$ diversities of each population in the Eastern cluster area. The three populations (A, B and $\mathrm{C})$ in the northeastern region of the Eastern cluster area exhibited the lowest diversities $(H<0.5$ and $\pi<0.001)$. The A population, which is the most northeastern population in the Eastern cluster area, showed the third-lowest $\pi$ value and a relatively low $H$ value. The two westernmost populations (I and J) exhibited low gene diversities $(H<0.5)$ but relatively high nucleotide diversities $(\pi \cong 0.002)$. The two central populations (D and E) and the southernmost population (G) in the Eastern cluster area showed high genetic diversities $(H>0.7, \pi>0.0024)$. Even though the F population had the largest sample size and the largest area, the genetic diversity (both $H$ and $\pi$ ) was relatively low. The four populations ( $\mathrm{K}$ to $\mathrm{N}$ ) in the Western cluster area exhibited intermediate diversities $(0.36<H<0.62$; $0.0013<\pi<0.0019)$. In the Southern cluster area, the $\mathrm{O}$ and $\mathrm{P}$ populations showed a large difference in nucleotide diversity, whereas the gene diversities were intermediate and similar.

We observed significant values $(P<0.05)$ in both $\mathrm{Fu}^{\prime} \mathrm{s}$ $F_{S}$ and Tajima's $D\left(F_{S}=-5.53 ; D=-1.56\right)$ and nonsignificance for the mismatch distribution test $(P>0.05)$ in the F population (Table 1). We observed the opposite trend in two northern populations, B and D: nonsignificant values in both Fu's $F_{S}$ and Tajima's D $(P>0.05)$ and significance for the mismatch distribution test $(P<0.05)$. In particular, the $B$ population had the largest Harpending's raggedness index value $(r=0.49)$ of all the populations. These results indicate expansion for the $F$ population and non-expansion for the $B$ and $D$ populations. The E population exhibited significance for both $\mathrm{Fu}^{\prime} \mathrm{s} \mathrm{F}_{\mathrm{S}}\left(\mathrm{F}_{\mathrm{S}}=-6.04, P<0.01\right)$ and the mismatch distribution test $(P<0.01)$. All six populations in the Western and Southern cluster areas exhibited nonsignificant indices for all tests.
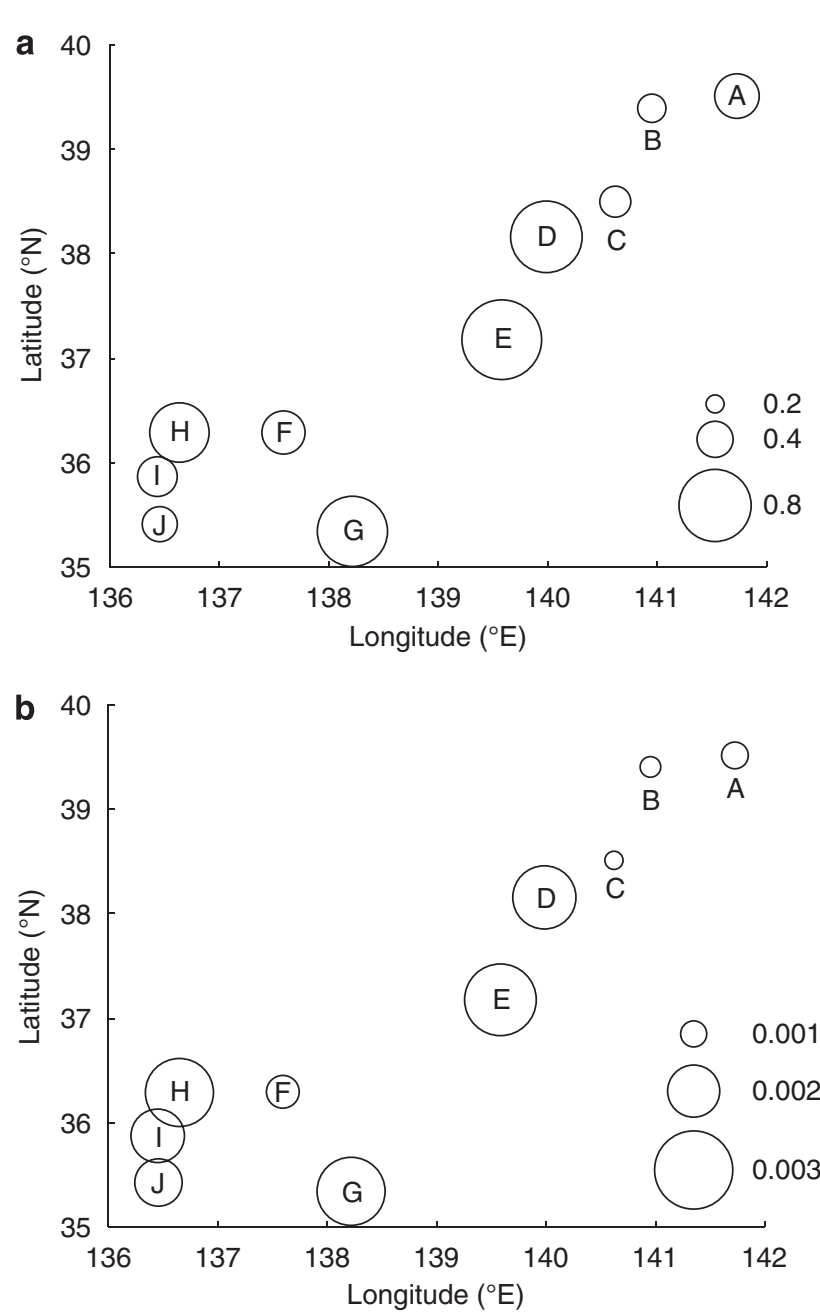

Figure 4 Relationships between geographical information (longitude and latitude) and the (a) gene and (b) nucleotide diversities of each population in the Eastern cluster area. The centre and the sizes of the open circles indicate the location and the magnitude of the diversity of each population, respectively.

\section{Discussion}

\section{Phylogeographic relationships}

We distinguished three lineages in Asian black bear populations by examining the haplotypes sampled from almost the entire range of the species in Japan. The border between the Western and Eastern cluster areas of the black bear appears to be Lake Biwa, which is approximately $100 \mathrm{~km}$ east of the border that separates two major lineages identified in Japanese macaques (Kawamoto et al., 2007), Japanese wild boar (Watanobe et al., 2003) and sika deer (Nagata et al., 1999). The distinct lineages in wild boar are thought to have resulted from multiple migrations of the species from the Asian Continent to Japan across land bridges that repeatedly formed between the Korean Peninsula and Kyushu Island during past glacial periods (Watanobe et al., 2003). In contrast, the distinct lineages in sika deer appear to have resulted from migration from the west across the Korea-Kyushu land bridge and from the north across the Sakhalin-Hokkaido-Honshu land bridge (Tamate et al., 1998; Nagata et al., 1999). 
The black bear is thought to have migrated from the Asian continent into Japan across the Korea-Kyushu land bridge 0.5 or $0.3 \mathrm{My} \mathrm{BP}$, or possibly in both periods (Dobson and Kawamura, 1998). Black bear lineages in Japan are very different from that in the Korean Peninsula, and haplotypes within the Asian continent and Taiwan were three times more divergent than those in Japan. In light of the uncertainty of molecular clock analysis (Ho et al., 2007) and the calibration points for bears, as well as the fallibility of the relationships among the three major lineages revealed by two phylogenetic analyses, we will not emphasize the time of divergence among the three black bear lineages in Japan. The $t_{\mathrm{MRCA}}$ analysis, however, suggests that the three lineages diverged during a short period of time. Given these results, it appears that black bears migrated into Japan once from the Asian continent, after which the three lineages diverged.

Given our data and the shift of vegetation in Japan that occurred during and between the glacial periods, we conclude that the expansion of Asian black bears across Japan took place as follows: First, the black bears that travelled to Kyushu Island across the Korea-Kyushu land bridge moved east towards the Japanese coast and the Honshu-Shikoku land bridge. Some haplotypes moved eastward to Lake Biwa, then expanded and diverged in the Eastern cluster area. Conversely, the black bears found along the Sea of Japan coast shifted to mountainous areas to follow the vegetation shift that took place during the interglacial period. Finally, some of the black bears that crossed the Honshu-Shikoku land bridge reached the southern Kinki area and became the ancient haplotypes of the Southern cluster.

\section{The effect of climatic oscillations on genetic structures in the Eastern cluster area}

We predicted that genetic diversity would be lower in the northern areas than in the southern areas due to the historical patterns of climatic oscillation. During the last glacial maximum, the distribution of broad-leaved forest was limited to south of approximately $38^{\circ} \mathrm{N}$, especially in the Kanto region (Tsukada, 1982). The distribution of the black bear may have changed to follow shifts in forest vegetation, causing a rapid expansion of the black bears northward after the end of the last glacial period. The resulting founder effect would thereby have reduced the genetic diversity in the northern areas.

Our results indicated that genetic diversity was generally lower north of approximately $38^{\circ} \mathrm{N}$, whereas it was relatively high in the middle of the Eastern cluster area (Figure 4), which corresponds with our initial prediction. However, other results do not. First, the three demographic tests (Table 1 ) rejected the expansion model for the B and D populations, which were both found north of $38^{\circ} \mathrm{N}$. Next, most of the observed haplotypes were locally distributed. If range expansion had occurred, the haplotypes observed in the expanded populations should also have been found in the ancestral population or populations, and as a result, would have become geographically more widespread (Templeton, 1998). However, we observed a wide distribution in two haplotypes (UtCR-E01 and UtCR-E06). These two haplotypes were at the core of the statistical parsimony network (Figure 3), which does not suggest a recent expansion from a particular population (Miller et al., 2006). Consequently, it is possible that UtCR-E01 and UtCR-E06 dispersed throughout the Eastern cluster area before the last glacial period, and their populations might have remained in northern refugia during that time.

Fossil pollen records suggest that mixed coniferous and deciduous broad-leaved forest occurred in the coastal regions south of approximately $38^{\circ} \mathrm{N}$ (Tsukada, 1982, 1985), whereas forests north of this latitude and mountainous areas in the middle of Honshu were dominated by boreal coniferous forest during the last glacial maximum. Although Hashimoto et al. (2003) reported that black bears prefer broad-leaved forest, they are omnivorous (Hashimoto, 2002) and also occur in coniferous forests in the cold and temperate zones of northeastern China (Xueliang, 1997). Moreover, pollen of Quercus (deciduous oaks) was occasionally observed between $38^{\circ} \mathrm{N}$ and $40^{\circ} \mathrm{N}$ during the last glacial maximum (Tsukada, 1983), indicating that small patches of deciduous broad-leaved forest existed north of $38^{\circ} \mathrm{N}$ during the last glacial maximum. Given this information, it is possible that the black bear inhabited northern refugia during the last glacial maximum. However, it is likely that any such populations were small given the limited resources that would have been available. Such a small population size may have caused genetic drift, thus explaining the decreased genetic diversity in the northeastern part of the Eastern cluster area.

Our results suggested demographic expansion for the $F$ population, and the genetic diversities in this population were low even though the population had the largest sample size and the largest area. Most of the area occupied by this population is high and mountainous, and the vegetation would have been tundra during the last glacial maximum (Tsukada, 1982). Therefore, no large population would have been maintained in the mountainous areas during the last glacial maximum, and bears would have gradually migrated into that area after the end of the last glacial period.

\section{The effect of climatic oscillations on genetic structures} in the Western and Southern cluster areas

All haplotypes in the Western cluster were unique to each population in females, and the divergence pattern of the haplotypes was geographically consistent (Ishibashi and Saitoh, 2004). These four populations in the Western cluster area are currently fragmented. The fragmentation might have begun several hundred years ago; thus, these populations would have originally been one continuous large population. Taking female-biased philopatry into account, haplotypes would have diverged locally from their ancestral type for a long time. Even though the area of evergreen broad-leaved forest would have decreased around the Western cluster area during glacial periods, deciduous broad-leaved forests would have replaced them in this area (Tsukada, 1982). Thus, such an alternative habitat would have maintained the black bear populations, and as a result, the genetic structure of black bears in the Western cluster area would not have been strongly affected by climatic oscillations during and between the glacial periods.

The Southern cluster area is composed of the $\mathrm{O}$ and $\mathrm{P}$ populations, which are largely or entirely surrounded by 
the sea. Shikoku was connected with Honshu and Kyushu during the glacial periods, thus these two populations would have been a single continuous population at some time in the past. We can explain how the genetic differences between these two populations were generated using a process similar to that which occurred in the Western cluster area. That is, the haplotypes diverged locally even though the populations were repeatedly connected, and then genetic diversity was lost due to reduced population sizes. We should also consider the alternative possibility that haplotypes diverged after these populations fragmented; however, this is unlikely given that the degree of divergence in the Southern cluster was not smaller than that in the Eastern and Western clusters.

Our data indicate that climatic oscillations during glacial periods exerted less influence on the genetic structure of the Western and Southern cluster area populations than on that of the Eastern cluster area populations. Even though the vegetation of the habitat changed from evergreen broad-leaved to deciduous broad-leaved forest (Tsukada, 1982), the change did not cause a shift in black bear distribution in the east-west direction, except for the area between Shikoku and southern Kinki. However, the sea between Honshu and Shikoku retreated to expose a land bridge during the last glacial maximum, and this bridge was an available habitat for black bears (Tsukada, 1982). It is likely that the Western and Southern cluster area populations expanded via the land bridge and that these populations mixed. Only one haplotype (UtCR-16) that was mainly observed in the $\mathrm{N}$ population was found in the $\mathrm{P}$ population, but no haplotype in the Southern cluster was detected in the Western cluster area. When we consider the current border between the Western cluster area and the Eastern cluster area, combined with female-biased philopatry, gene flow mediated by females between the Western cluster area and the Southern cluster area would be rare. Therefore, the frequency of haplotypes entering from other areas would be low, and diversity could have been lost as a result of genetic drift due to recent decreases in population size.

\section{Conclusion}

We detected three lineages in the mtDNA haplotypes of the Asian black bear in Japan, and they appear to have diverged soon after migration of the species into Japan. Haplotypes of the Eastern cluster were distributed in central and eastern Honshu. Our results suggest that the ancestral types expanded first and then diverged into local haplotypes. During repeated glacial periods, the black bear could not inhabit high mountainous areas, but small populations would have remained in refugia in northeastern Honshu. As a result, genetic diversities decreased because of genetic drift. The black bear's distribution expanded after the end of the last glacial period, but genetic diversity remained low. In contrast, the genetic structure of black bear populations in western and southern Japan was not strongly influenced by the climatic oscillations that took place during the Quaternary Era. The extant black bear populations are fragmented, but they would have been continuous in the past. Haplotypes would have diverged locally, and the local genetic structures that were generated over long periods would have been maintained. However, current reductions in population sizes due to recent habitat fragmentation will ultimately decrease the genetic diversity of Asian black bears in Japan.

\section{Acknowledgements}

We thank $\mathrm{T}$ Saitoh for encouragement throughout the study, as well as T Shimada, Y Segawa and S Ookubo for their support in the preparation of samples and Y Shimada, K Hirayama and F Kitamura for their support with the laboratory work. H Takahara provided advice on the shift of vegetation. Helpful comments on our article were provided by FE Zachos and two anonymous referees. T Tsubota, S Nakamura, T Maruyama, R Kishimoto, T Ohba, Y Yoneda, E Nozaki, T Hayashi, Y Yoshida, S Saito, H Nakayama, H Higashi, K Inoue, Y Kato, A Hayashi, Y Nagakubo and the Shikoku Institute of National History contributed samples. We also thank the local governments and the branches of the Japan Hunting Association for collecting samples in the following prefectures: Iwate, Yamagata, Niigata, Toyama, Ishikawa, Fukui, Yamanashi, Gunma, Nagano, Shizuoka, Shiga and Nara. This study was partially supported by a Grant-in-Aid for Scientific Research (no. 20380098) from the Ministry of Education, Culture, Sports, Science and Technology, Japan, and by the Pollution Control Research Fund of the Ministry of the Environment, Japan.

\section{References}

Biodiversity Center of Japan (2004). Mammalia, Vol. 1 Biodiversity Center of Japan: Fujiyoshida.

Clement M, Posada D, Crandall KA (2000). TCS: a computer program to estimate gene genealogies. Mol Ecol 9: 1657-1659.

Coulon A, Guillot G, Cosson JF, Angibault JMA, Aulagnier S, Cargnelutti B et al. (2006). Genetic structure is influenced by landscape features: empirical evidence from a roe deer population. Mol Ecol 15: 1669-1679.

Dobson M, Kawamura Y (1998). Origin of the Japanese land mammal fauna: allocation of extant species to historicallybased categories. Quat Res 37: 385-395.

Drummond AJ, Rambaut A (2007). BEAST: Bayesian evolutionary analysis by sampling trees. BMC Evol Biol 7: 1-8.

Eisen JA (1999). Mechanistic basis for microsatellite instability. In: Goldstein DB, Schlötterer C (eds). Microsatellites: Evolution and Applications. Oxford University Press: Oxford, UK, pp 34-48.

Excoffier L, Laval G, Schneider S (2005). Arlequin ver. 3.0: an integrated software package for population genetics data analysis. Evol Bioinform Online 1: 47-50.

Excoffier L, Smouse PE, Quattro JM (1992). Analysis of molecular variance inferred from metric distances among DNA haplotypes: application to human mitochondrial DNA restriction data. Genetics 131: 479-491.

Fu YX (1997). Statistical tests of neutrality of mutations against population growth, hitchhiking and background selection. Genetics 147: 915-925.

Fujii N, Tomaru N, Okuyama K, Koike T, Mikami T, Ueda K (2002). Chloroplast DNA phylogeography of Fagus crenata (Fagaceae) in Japan. Plant Syst Evol 232: 21-33.

Guillot G, Estoup A, Mortier F, Cosson JF (2005). A spatial statistical model for landscape genetics. Genetics 170: 1261-1280.

Hashimoto Y (2002). Seasonal food habits of the Asiatic black bear (Ursus thibetanus) in the Chichibu Mountains, Japan. Mammal Study 27: 65-72. 
Hashimoto Y, Kaji M, Sawada H, Takatsuki S (2003). Five-year study on the autumn food habits of the Asiatic black bear in relation to nut production. Ecol Res 18: 485-492.

Hewitt G (2000). The genetic legacy of the Quaternary ice ages. Nature 405: 907-913.

Ho SYW, Shapiro B, Phillips MJ, Cooper A, Drummond AJ (2007). Evidence for time dependency of molecular rate estimates. Syst Biol 56: 515-522.

Hou W-R, Chen Y, Wu X, Hu J-C, Peng Z-S, Yang J et al. (2007). A complete mitochondrial genome sequence of Asian black bear Sichuan subspecies (Ursus thibetanus mupinensis). Int J Biol Sci 3: 85-90.

Hwang D-S, Ki J-S, Jeong D-H, Kim B-H, Lee B-K, Han S-H et al. (2008). A comprehensive analysis of three Asiatic black bear mitochondrial genomes (subspecies ussuricus, formosanus and mupinensis), with emphasis on the complete mtDNA sequence of Ursus thibetanus ussuricus (Ursidae). Mitochondrial DNA 19: 418-429.

Ishibashi Y, Saitoh T (2004). Phylogenetic relationships among fragmented Asian black bear (Ursus thibetanus) populations in western Japan. Conserv Genet 5: 311-323.

Jobb G, von Haeseler A, Strimmer K (2004). TREEFINDER: a powerful graphical analysis environment for molecular phylogenetics. BMC Evol Biol 4: 18.

Kanno M, Yokoyama J, Suyama Y, Ohyama M, Itoh T, Suzuki M (2004). Geographical distribution of two haplotypes of chloroplast DNA in four oak species (Quercus) in Japan. J Plant Res 117: 311-317.

Kawamoto Y, Shotake T, Nozawa K, Kawamoto S, Tomari K, Kawai S et al. (2007). Postglacial population expansion of Japanese macaques (Macaca fuscata) inferred from mitochondrial DNA phylogeography. Primates 48: 27-40.

Li W-H (1977). Distribution of nucleotide differences between two randomly chosen cistrons in a finite population. Genetics 85: 331-337.

Matsuhashi T, Masuda R, Mano T, Murata K, Aiurzaniin A (2001). Phylogenetic relationships among worldwide populations of the brown bear Ursus arctos. Zoolog Sci 18: 1137-1143.

Matsuhashi T, Masuda R, Mano T, Yoshida MC (1999). Microevolution of the mitochondrial DNA control region in the Japanese brown bear (Ursus arctos) population. Mol Biol Evol 16: 676-684.

Miller CR, Waits LP, Joyce P (2006). Phylogeography and mitochondrial diversity of extirpated brown bear (Ursus arctos) populations in the contiguous United States and Mexico. Mol Ecol 15: 4477-4485.

Minaki M (1996). Distributional change of Fagus crenata during the Quaternary period. Jpn J Ecol 46: 171-174 (in Japanese with English abstract).

Nagata J, Masuda R, Tamate HB, Hamasaki S, Ochiai K, Asada $\mathrm{M}$ et al. (1999). Two genetically distinct lineages of the sika deer, Cervus nippon, in Japanese islands: comparison of mitochondrial D-loop region sequences. Mol Phylogenet Evol 13: $511-519$

Nixon KC (1999). The parsimony ratchet, a new method for rapid parsimony analysis. Cladistics 15: 407-414.

Ohi T, Wakabayashi M, Wu S, Murata J (2003). Phylogeography of Stachyurus praecox (Stachyuraceae) in the Japanese Archipelago based on chloroplast DNA haplotypes. J Jpn Bot 78: 1-14

Oka T, Miura S, Masaki T, Suzuki W, Osumi K, Saitoh S (2004). Relationship between changes in beechnut production and Asiatic black bears in northern Japan. J Wildl Manage 68: 979-986.
Oshida T, Ikeda K, Yamada K, Masuda R (2001). Phylogeography of the Japanese giant flying squirrel, Petaurista leucogenys, based on mitochondrial DNA control region sequences. Zoolog Sci 18: 107-114.

Posada D, Crandall KA (1998). MODELTEST: testing the model of DNA substitution. Bioinformatics 14: 817-818.

Rogers AR, Harpending H (1992). Population growth makes waves in the distribution of pairwise genetic differences. Mol Biol Evol 9: 552-569.

Sambrook J, Fritsch EF, Maniatis T (1989). Molecular Cloning: A Laboratory Manual, 2nd edn. Cold Spring Harbor Laboratory Press: Cold Spring Harbor, NY.

Schneider S, Excoffier L (1999). Estimation of past demographic parameters from the distribution of pairwise differences when the mutation rates vary among sites: application to human mitochondrial DNA. Genetics 152: 1079-1089.

Servheen C (1990). The status and conservation of the bears of the world. International Conference on Bear Research and Management, Vol. Monograph Series 2.

Slatkin M, Hudson RR (1991). Pairwise comparisons of mitochondrial DNA sequences in stable and exponentially growing populations. Genetics 129: 555-562.

Tajima F (1989). Statistical method for testing the neutral mutation hypothesis by DNA polymorphism. Genetics 123 585-595.

Tamate HB, Tatsuzawa S, Suda K, Izawa M, Doi T, Sunagawa K. et al. (1998). Mitochondrial DNA variation in local populations of the Japanese sika deer, Cervus nippon. J Mammal 79: 1396-1413.

Templeton AR (1998). Nested clade analyses of phylogeographic data: testing hypotheses about gene flow and population history. Mol Ecol 7: 381-397.

Tomaru N, Takahashi M, Tsumura Y, Takahashi M, Ohba K (1998). Intraspecific variation and phylogeographic patterns of Fagus crenata (Fagaceae) mitochondrial DNA. Am J Bot 85: 629-636.

Tsai C-L, Chou Y-C, Shih C-C, Cheng H-C, Yang C-C, Kao H-W (2009). The complete mitochondrial genome of the Formosan black bear (Ursus thibetanus formosanus). Zootaxa 1971: 50-58.

Tsukada M (1982). Cryptomeria japonica: glacial refugia and late-glacial and postglacial migration. Ecology 63 1091-1105.

Tsukada M (1983). Vegetation and climate during the last glacial maximum in Japan. Quat Res 19: 212-235.

Tsukada M (1985). Map of vegetation during the last glacial maximum in Japan. Quat Res 23: 369-381.

Vos RA (2003). Accelerated likelihood surface exploration: the likelihood ratchet. Syst Biol 52: 368-373.

Waits LP, Sullivan J, O'Brien SJ, Ward RH (1999). Rapid radiation events in the family Ursidae indicated by likelihood phylogenetic estimation from multiple fragments of mtDNA. Mol Phylogenet Evol 13: 82-92.

Watanobe T, Ishiguro N, Nakano M (2003). Phylogeography and population structure of the Japanese wild boar Sus scrofa leucomystax: mitochondrial DNA variation. Zoolog Sci 20: 1477-1489.

Xueliang X (1997). Historical and present status of the Asiatic black bear in northeast China. Bears: Their Biology and Management 9: 53-55.

Yu L, Li Y-W, Ryder O, Zhang Y-P (2007). Analysis of complete mitochondrial genome sequences increases phylogenetic resolution of bears (Ursidae), a mammalian family that experienced rapid speciation. BMC Evol Biol 7: 198. 